
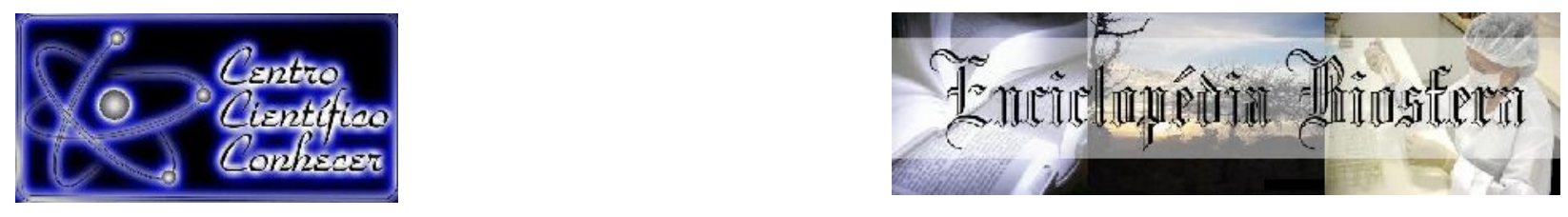

\title{
INFLUÊNCIA DA CONCENTRAÇÃO E DO TEMPO DE SEDIMENTAÇÃO NO USO DE Abelmoschus esculentus (L.) MOENCH (QUIABO) COMO AUXILIAR DE FLOCULAÇÃO
}

Heloise Beatriz Quesada ${ }^{1}$ Laís Gimenes Vernasqui ${ }^{2}$ Renata Mariane de Souza ${ }^{1}$ Guilherme Gobbi Teixeira ${ }^{3}$ Flávia Vieira da Silva Medeiros ${ }^{4}$

${ }^{1}$ Mestranda do Programa de Pós-Graduação em Engenharia Química da Universidade Estadual de Maringá, Campus Maringá, Brasil.

(heloisequesada@gmail.com)

${ }^{2}$ Mestranda do Programa de Pós-Graduação em Engenharia e Tecnologia Espaciais do Instituto Nacional de Pesquisas Espaciais, Campus São José dos Campos, Brasil.

${ }^{3}$ Mestrando em Tecnologia Ambiental do Instituto Politécnico de Bragança, Campus Bragança, Portugal.

${ }^{4}$ Docente da Universidade Tecnológica Federal do Paraná - Campus Campo Mourão, Brasil. Departamento de Ambiental.

Recebido em: 06/04/2019 - Aprovado em: 10/06/2019 - Publicado em: 30/06/2019 DOI: 10.18677/EnciBio_2019A181 utilização de polímeros químicos, que causam impactos ambientais. Com isso, os polímeros naturais estão sendo pesquisados, como auxiliares na floculação ou mesmo como coagulantes principais, devido a sua biodegradabilidade. Desta forma, o objetivo deste trabalho foi verificar a influência da concentração e do tempo de sedimentação no uso do quiabo como auxiliar de floculação em conjunto com o coagulante químico Policloreto de Alumínio (PAC). Para isso, determinou-se primeiramente o $\mathrm{pH}$ ideal da água. Posteriormente, a fim de avaliar a influência do quiabo para o tratamento, realizaram-se testes apenas com PAC, variando a sua concentração (0-10 ppm) e com a combinação de coagulante (2 ppm) e quiabo, variando de 0,5 a 3 ppm. Os parâmetros analisados foram cor, turbidez, COD e UV$254 \mathrm{~nm}$, medidos após diferentes tempos de sedimentação. Foi verificado que a concentração ótima de PAC foi de 6 ppm, que removeu $89,82 \%$ da cor e $94,24 \%$ da turbidez. Já no ensaio do conjunto PAC/quiabo a concentração ótima de quiabo foi de 0,5 ppm, que removeu $82,51 \%$ de cor e $87,82 \%$ de turbidez. Não foi verificada necessidade de tempos de sedimentação maiores que cinco minutos. Mesmo não superando o tratamento com 6ppm de PAC, comparando com o ensaio com 2 ppm de PAC sem a adição do auxiliar, notou-se a relevância do quiabo para o tratamento, já que o primeiro apresentou somente 76,7 e $81,76 \%$ de remoção de cor e turbidez, respectivamente.

PALAVRAS-CHAVE: floculação, polímero natural, quiabo. 


\title{
INFLUENCE OF CONCENTRATION AND SEDIMENTATION TIME IN THE USE OF Abelmoschus esculentus (L.) MOENCH (OKRA) AS FLOCCULATION AUXILIARY
}

\begin{abstract}
The conventional treatment of drinking water is carried out with the use of chemical polymers, which cause environmental impacts. Thus, natural polymers are being investigated as flocculation auxiliaries or even as major coagulants because of their biodegradability. Thus, the objective of this work was to verify the influence of concentration and sedimentation time on the use of okra as a flocculation auxiliary of the chemical coagulant Poly Aluminum Chloride (PAC). For this, the water ideal pH was first determined. Subsequently, in order to evaluate the influence of okra on the treatment, tests were performed with PAC only, varying its concentration $(0-10 \mathrm{ppm})$ and with the combination of coagulant (2 ppm) and okra, ranging from 0.5 at $3 \mathrm{ppm}$. The analyzed parameters were color, turbidity, COD and UV-254 nm, measured after different sedimentation times. It was found that the optimum PAC concentration was $6 \mathrm{ppm}$, which removed $89,82 \%$ of the color and $94.24 \%$ of the turbidity. In the PAC / okra test, the optimum okra concentration was $0.5 \mathrm{ppm}$, which removed $82.51 \%$ of color and $87.82 \%$ of turbidity. There was no need for sedimentation times greater than 5 minutes. Although not exceeding the 6ppm PAC treatment, comparing with the 2 ppm PAC test without the addition of the auxiliary, the relevance of the okra was noted for the treatment, since the former presented only 76.7 and $81,76 \%$ of color removal and turbidity, respectively.
\end{abstract}

KEYWORDS: flocculation, natural polymer, okra.

\section{INTRODUÇÃO}

A poluição das águas resulta das diversas atividades humanas, incluindo industriais, domésticas e agricultura. Essa poluição não é devida somente a derivados do petróleo e despejos orgânicos, mas também por substâncias químicas, como corantes, fertilizantes e pesticidas (CRINI; BADOT, 2007). Quando há a poluição, o tratamento de água se torna necessário (RENAULT et al., 2009).

O tratamento convencional de água, dentre os métodos mais conhecidos e utilizados, consiste nas etapas de coagulação/floculação. Esse método é o mais empregado devido ao baixo custo de operação, visto que as duas etapas são consideradas eficientes na remoção de coloides, partículas suspensas e dissolvidas. Essa remoção acontece devido à agregação induzida de partículas e posterior sedimentação (OLADOJA, 2015).

Sais de alumínio são os coagulantes mais utilizados em conjunto com polímeros orgânicos. Esses coagulantes são, na maioria das vezes, de alto custo e, em vários países, é necessária a importação. Apesar de seu amplo emprego, são sensíveis a variações de $\mathrm{pH}$, de temperatura, além de serem requeridos volumes consideráveis para tratamento efetivo e produzirem grande quantidade de lodo (SHARMA et al., 2006; BRATBY, 2016). Para o ambiente, o maior problema relacionado à utilização de sais de alumínio e polímeros sintéticos é a não biodegradabilidade.

Em substituição a esses polímeros sintéticos, os polímeros naturais vêm sendo utilizados em diversas pesquisas científicas, exercendo papel de coagulante ou auxiliar de floculação. Segundo Renault et al. (2009), polímeros naturais são mais 
vantajosos em relação aos polímeros inorgânicos pois produzem flocos mais compactos e densos, além de formarem menores volumes de lodo no tratamento.

Dessa maneira, quando usados em conjunto com o coagulante químico, esses polímeros podem reduzir o volume do lodo e do alumínio e promover a mesma eficiência de remoção de particulados e matéria orgânica. Portanto, causam menos impactos ao meio ambiente, tanto em relação à biodegradabilidade, quanto à toxicidade (CHUNG et al., 2018).

Dentre os polímeros naturais utilizados como floculantes no tratamento de água, encontra-se o quiabo (Abelmoschus esculentus (L.) Moench.). Estudos prévios já avaliaram sua eficiência como coagulante e floculante natural no tratamento de água e efluentes (LEE et al., 2015; FREITAS et al., 2015; CHUNG et al., 2018).

Considerando o exposto, o objetivo do presente estudo foi avaliar a influência da concentração e do tempo de sedimentação no uso de Abelmoschus esculentus (L.) Moench como auxiliar de floculação em conjunto com o coagulante químico Policloreto de Alumínio (PAC), a fim de verificar as melhores condições em relação à concentração e tempo de sedimentação.

\section{MATERIAL E MÉTODOS}

Os experimentos foram realizados no Núcleo de Pesquisa em Engenharia Ambiental (NUPEA) da Universidade Tecnológica Federal do Paraná - Campus Campo Mourão.

\section{Preparo das soluções}

A solução do coagulante químico PAC foi preparada em balão volumétrico na proporção de 1:100, utilizando água destilada para completar o volume. A solução estoque de quiabo, já seco e triturado, foi preparada na proporção de 1:100, também utilizando água destilada para completar o volume. Dessa solução estoque, foi realizada novamente uma diluição 1:100. O alcalinizante utilizado para correção do $\mathrm{pH}$ da água bruta foi o $\mathrm{Ca}(\mathrm{OH})_{2}$, sendo o preparo realizado em balão volumétrico na proporção de 1:100.

\section{Escolha do pH}

Para determinação do $\mathrm{pH}$ ideal da água bruta para o tratamento com o floculante extraído do quiabo, um ensaio preliminar foi realizado, em que quatro valores de $\mathrm{pH}:$ 7, 8, 9 e 10 foram avaliados, e as concentrações de PAC e quiabo foram fixadas em, respectivamente, 10 e 3 ppm. Ao término da etapa de coagulação e floculação, as amostras foram deixadas em repouso por 15 minutos e tiveram a turbidez determinada. $\mathrm{O}$ valor de $\mathrm{pH}$ utilizado no ensaio que apresentou menor turbidez remanescente foi escolhido para ser utilizada nos ensaios posteriores.

\section{Ensaios de coagulação/floculação utilizando PAC como coagulante e solução de quiabo como floculante}

Foram realizados ensaios de coagulação e floculação em equipamento Jar Test de seis hastes e com velocidade regulável (modelo 218/LDB, Nova Ética, Brasil). Para a coagulação, a velocidade utilizada foi de $120 \mathrm{rpm}$ durante um minuto, enquanto para a floculação, a velocidade foi diminuída para $60 \mathrm{rpm}$ e mantida durante 15 minutos. 
Ao término das etapas de coagulação e floculação, o equipamento era desligado e amostras eram retiradas de cada jarro conforme o tempo de sedimentação predefinido para medição de cor, turbidez, COD e UV-254 nm. Os tempos de sedimentação utilizados nesses ensaios foram de: 0, 5, 10, 15, 30, 45 e 60 minutos.

Para verificar a influência do auxiliar de floculação, foram realizados ensaios somente com PAC e também com PAC combinado com a solução de quiabo. Nos ensaios que utilizaram somente 0 coagulante químico para 0 tratamento, a concentração de PAC variou de 0 a $10 \mathrm{ppm}$. Já nos ensaios com o quiabo, a concentração do coagulante foi mantida fixa em 2 ppm e a concentração de quiabo variou de 0,5 a $3 \mathrm{ppm}$. A solução do floculante era adicionada aos jarros quatro minutos após o início da floculação.

\section{RESULTADOS E DISCUSSÃO}

\section{Escolha do $\mathrm{pH}$}

A Tabela 1 apresenta a turbidez residual e o desvio padrão (DP) após tratamento com os diferentes valores de $\mathrm{pH}$ iniciais.

TABELA 1: Resultados dos ensaios para determinação do $\mathrm{pH}$ ideal

\begin{tabular}{ccc}
\hline Faixa de $\mathbf{~ H H}$ & Turbidez média (uT) & DP da turbidez \\
\hline 7 & 4,255 & 0,629325 \\
8 & 3,000 & 0,282843 \\
9 & 2,645 & 0,332340 \\
10 & 2,975 & 0,035355 \\
\hline
\end{tabular}

Como o menor valor de turbidez foi resultado do tratamento com $\mathrm{pH} 9$, este foi o valor escolhido para os ensaios posteriores.

\section{Ensaios de coagulação/floculação utilizando Policloreto de Alumínio (PAC)}

Os resultados obtidos nos ensaios realizados somente com o coagulante químico PAC estão representados na Figura 1. A água bruta apresentou $139 \mathrm{uC}$ de cor, 61,8 uT de turbidez, $0,21 \mathrm{~cm}^{-1}$ de UV-254 nm e 102,77 $\mathrm{mg} \mathrm{L}^{-1}$ de Carbono Orgânico Dissolvido (COD). 

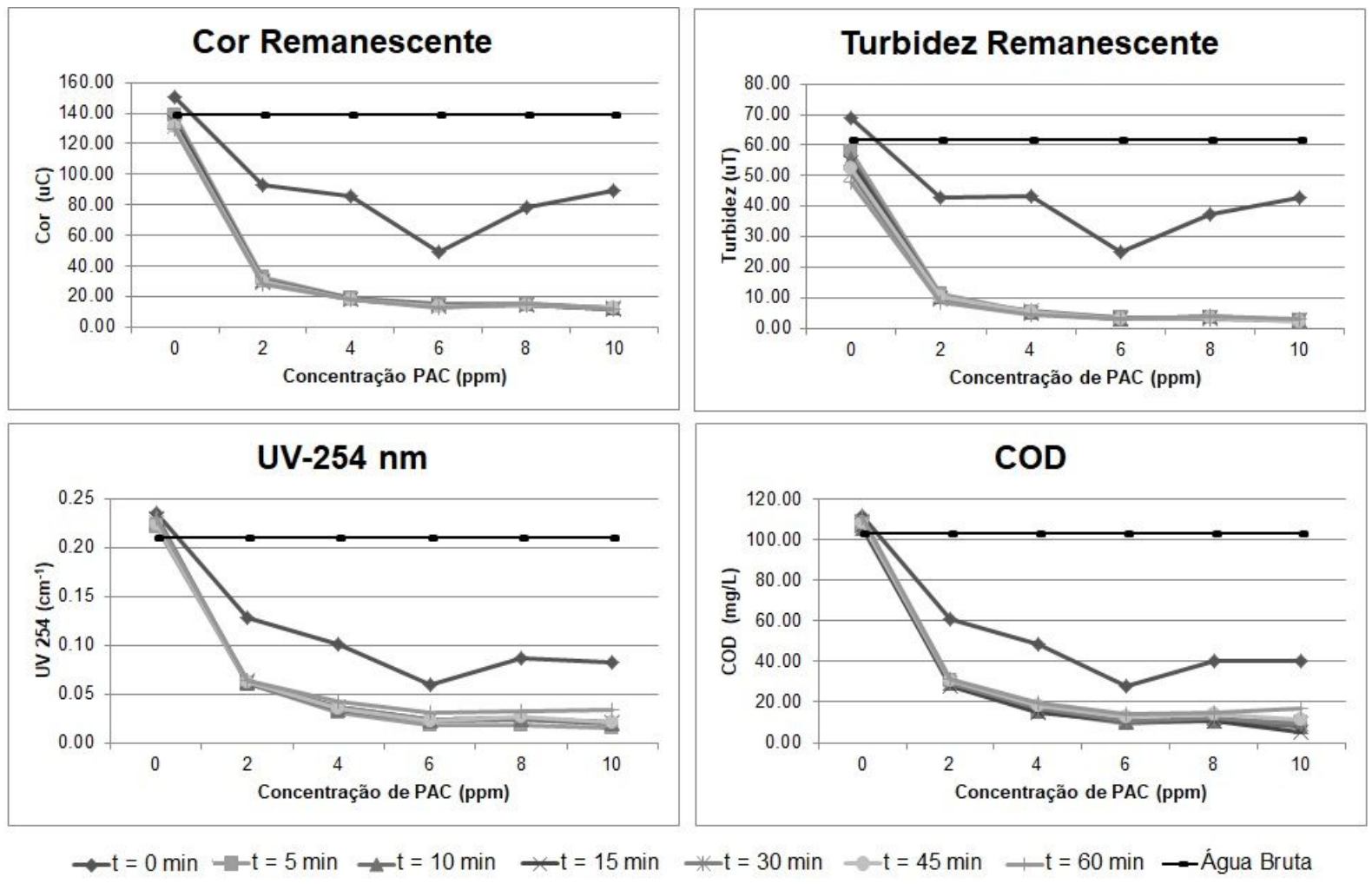

FIGURA 1: Cor, turbidez, UV-254 nm e Carbono Orgânico Dissolvido (COD) após coagulação/floculação utilizando Policloreto de Alumínio (PAC) em diferentes tempos de sedimentação.

A partir da Figura 1, foi possível observar que os parâmetros avaliados sofreram uma redução significativa quando a concentração de PAC passou de 0 para 2 ppm e continuaram diminuindo até a aplicação de 6 ppm do coagulante. A partir dessa concentração, não se observou uma diminuição significativa dos parâmetros com o aumento da concentração de PAC, que variou até 10 ppm. Como observado para a cor, que após cinco minutos de sedimentação de 139 para 32,4 uC após o aumento de PAC de 0 para 2 ppm. Com o aumento para 6 ppm de coagulante químico, a cor ainda sofreu redução significativa, alcançando 14,15 uC, porém, com $8 \mathrm{ppm}$, a cor remanescente foi de 14,05 uC, diferenciando-se apenas $0,7 \%$ do valor obtido com 6 ppm.

Analisando a Figura 1, observou-se que a curva que representa o tempo de sedimentação igual a 0 minutos se diferencia das demais pois as partículas ainda se encontravam em suspensão no momento da coleta das amostras. A partir de cinco minutos, as curvas se aproximaram, o que indica que, quando a água é tratada apenas com o coagulante químico, um tempo de sedimentação igual a cinco minutos resulta em reduções nos parâmetros muito semelhantes às que ocorrem com tempos maiores.

Em resumo, considera-se como melhor condição de tratamento a aplicação de 6 ppm de PAC e o tempo de sedimentação igual a cinco minutos, combinação que resultou em valores de cor, turbidez, UV-254 nm e COD de 14,15 uC, 3,56 uT, $0,02 \mathrm{~cm}^{-1}$ e $9,37 \mathrm{mg} \mathrm{L}^{-1}$, respectivamente. Em termos de remoção, nessas condições, a cor e turbidez sofreram redução de, respectivamente, 89,82 e 94,24\%. 
Yarahmadi et al. (2009), obtiveram turbidez residual de, aproximadamente, 7 uT após coagulação/floculação com 10 ppm de PAC, 30 minutos de sedimentação e $\mathrm{pH}$ 8. Esse resultado foi inferior ao encontrado no tratamento realizado no presente trabalho, em que a turbidez residual foi de 2,53 uT com a mesma concentração e tempo de sedimentação. $O$ fato pode ser justificado pelas melhores condições operacionais de mistura rápida e lenta, visto que os autores utilizaram 120 rpm e 40 rpm por 20 minutos, respectivamente. Na melhor condição de tratamento citada anteriormente, a turbidez residual já era inferior ao valor encontrado pelos autores.

Ensaios de coagulação/floculação utilizando Abelmoschus esculentus (L.) Moench. (Quiabo) como auxiliar de floculação em conjunto com o Policloreto de Alumínio (PAC)

A fim de comparar o comportamento do tratamento utilizando somente PAC com o tratamento utilizando quiabo como auxiliar de floculação, os ensaios seguintes foram realizados com a menor concentração de PAC, no caso, 2 ppm. A Figura 2 representa os resultados obtidos nestes ensaios.
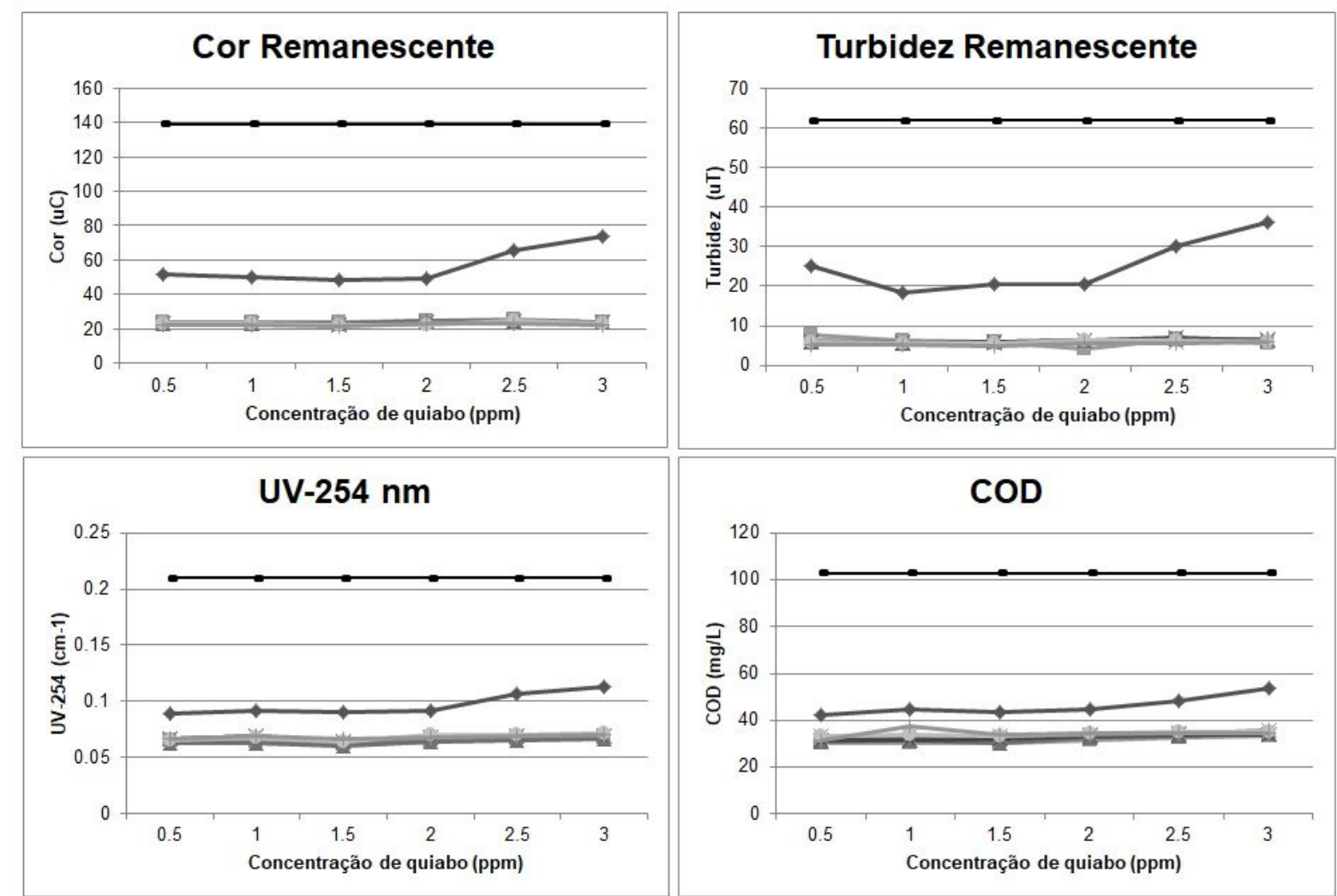

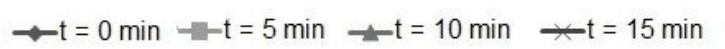

* $\mathrm{t}=30 \mathrm{~min} \longrightarrow \mathrm{t}=45 \mathrm{~min} \leftarrow \mathrm{t}=60 \mathrm{~min} \longrightarrow$ Água Bruta

FIGURA 2: Cor, turbidez, UV-254 nm e Carbono Orgânico Dissolvido (COD) após coagulação/floculação utilizando Policloreto de Alumínio (PAC) a $2 \mathrm{ppm}$ e Abelmoschus esculentus (L.) Moench como auxiliar de floculação em diferentes tempos de sedimentação, em minutos.

A partir da Figura 2 foi possível observar que a utilização do PAC em conjunto com o auxiliar de floculação quiabo promoveu redução significativa nos 
parâmetros analisados. Ainda, observou-se que as diferentes curvas que representam os tempos de sedimentação se comportaram de maneira semelhante: não oscilaram de forma significativa conforme o aumento da concentração de quiabo, o que indicou que concentrações de $0,5 \mathrm{ppm}$ são suficientes para o tratamento.

Novamente, as remoções dos parâmetros avaliados não aumentaram significativamente com tempos de sedimentação maiores do que 5 minutos, não sendo, portanto necessário utilizar tempos maiores para o tratamento com o PAC em conjunto com o auxiliar de floculação.

O tempo de sedimentação reduzido pode ser uma das maiores vantagens do uso do quiabo como floculante natural, principalmente quando comparados com resultados obtidos anteriormente em trabalhos que a Moringa oleifera Lam foi utilizada como coagulante natural. Theodoro et al. (2015), que avaliaram os tempos de sedimentação de 1 a 60 min após coagulação/floculação realizada pelo coagulante extraído de sementes de Moringa oleifera Lam e observaram maior remoção de cor em 40 e 60 min de sedimentação. Da mesma forma, Cardoso et al. (2008), avaliaram os tempos de decantação de 60, 90 e 120 min, observando que de forma geral, quanto maior o tempo, maior a remoção de cor e turbidez.

Vale ressaltar que o extrato do quiabo foi utilizado como auxiliar de floculação, e não como coagulante principal, como no caso dos autores citados anteriormente. Portanto, a diferença encontrada se deve ao papel da solução de Abelmoschus esculentus (L.) Moench, que foi adensar os flocos, reduzindo assim o tempo de sedimentação.

Valverde et al. (2013), utilizaram cloreto férrico como coagulante e Moringa oleifera Lam. como auxiliar, verificando que é possível reduzir o tempo de sedimentação para 15 min com alta eficiência de remoção de cor e turbidez. Os autores observaram as melhores condições de operação também com 15 min de sedimentação, utilizando PAC como coagulante e Moringa oleifera Lam. como auxiliar. Tendo em vista os resultados apresentados, o tratamento com Abelmoschus esculentus (L.) Moench como auxiliar de floculação requer menor tempo de sedimentação, mais especificamente, 5 min, em comparação com as sementes de Moringa oleifera Lam.

Estes resultados comprovam que o uso de um polímero orgânico como auxiliar de coagulação/floculação, o desempenho da floculação é aperfeiçoado, sendo que sua associação ao coagulante permite reduzir o tempo de sedimentação (BRATBY, 2016).

Em resumo, consideraram-se as condições ótimas de tratamento: 2 ppm de PAC, 0,5 ppm de quiabo e 5 minutos de tempo de sedimentação, que resultaram em valores de cor, turbidez, COD e UV-254 de, respectivamente, 24,3 uC, 7,5 uT, 30,38 $\mathrm{mg} \mathrm{L}^{-1}$ e $0,065 \mathrm{~cm}^{-1}$. Em termos de remoção, os parâmetros cor e turbidez sofreram redução de 82,51 e 87,82\%, respectivamente, nessas condições.

A fim de observar o efeito da adição de quiabo, foram construídos gráficos de comparação entre os ensaios com as melhores concentrações encontradas, ou seja, o ensaio em que se utilizou 6 ppm de PAC e o conjunto de 0,5 ppm de quiabo a 2 ppm de PAC, além dos dados do tratamento apenas com 2 ppm de PAC (Figura $3)$. 

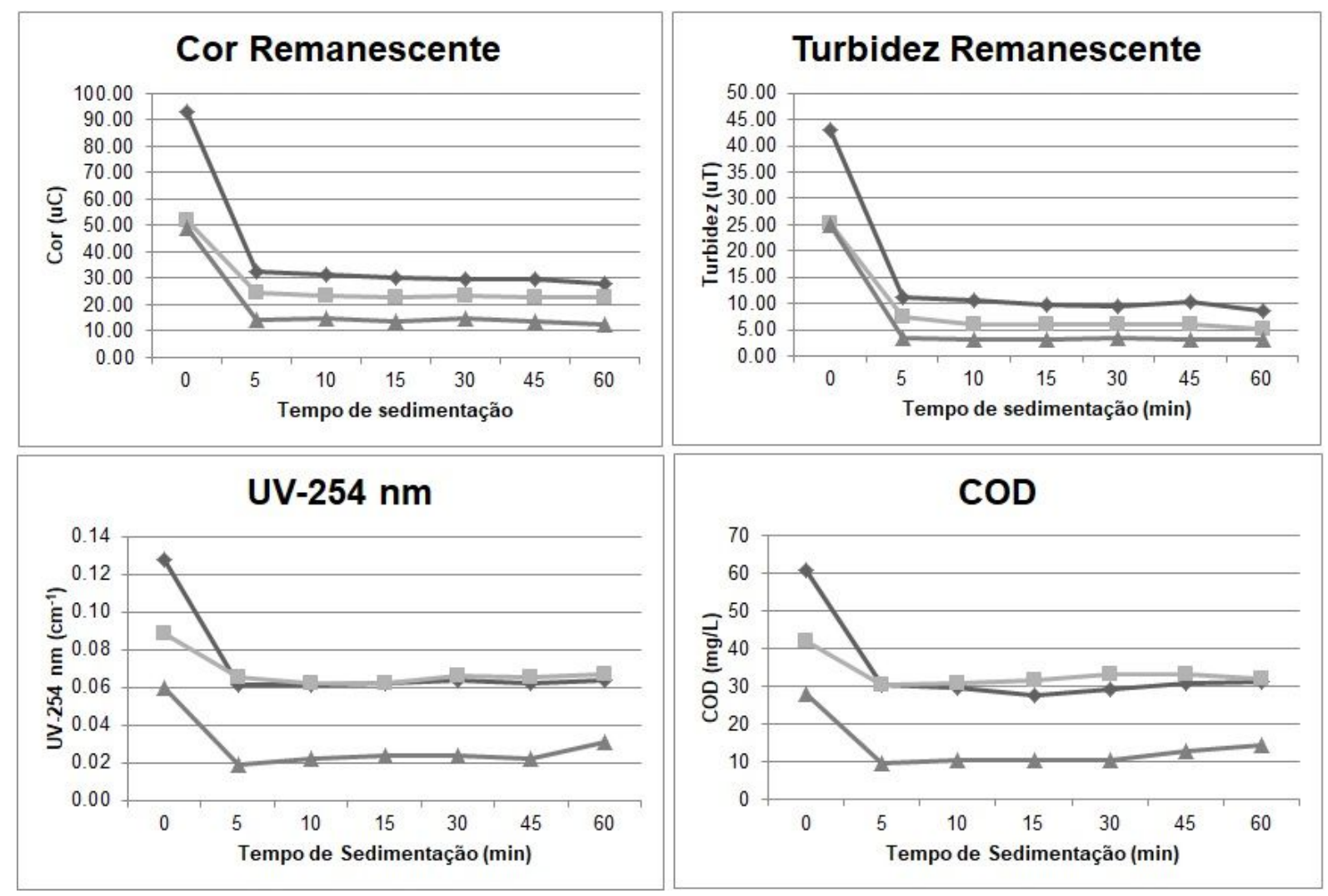

$\multimap 2 \mathrm{ppm}$ de PAC $-2 \mathrm{mpm}$ de PAC $+0,5 \mathrm{ppm}$ de quiabo $\rightarrow 6 \mathrm{ppm}$ de PAC

FIGURA 3: Cor, turbidez, UV-254 nm e Carbono Orgânico Dissolvido (COD) após coagulação/floculação utilizando: Policloreto de Alumínio (PAC) a 2 ppm, PAC a 6 ppm e PAC a 2 ppm com a adição de 0,5 ppm de Abelmoschus esculentus (L.) Moench., em diferentes tempos de sedimentação, em minutos.

Comparando a curva "6 ppm de PAC", concentração ótima encontrada, com a "2 ppm de PAC e 0,5 ppm de Quiabo", foi possível notar que esses resultados não superaram os encontrados no tratamento com 6 ppm de PAC. Este resultou em valores de cor, turbidez, UV-254 nm e COD de 14,15 uC, 3,56 uT, 0,02 cm e 9,37 $\mathrm{mg} \mathrm{L}^{-1}$, respectivamente, enquanto aquele resultou em $24,3 \mathrm{uC}, 7,5 \mathrm{uT}, 0,065 \mathrm{~cm}^{-1} \mathrm{e}$ $30,38 \mathrm{mg} \mathrm{L}^{-1}$, respectivamente.

Porém, comparando as curvas "2 ppm de PAC" e a relativa à adição de quiabo, observou-se que nos parâmetros cor e turbidez, houve maior redução quando adicionado o quiabo como auxiliar de floculação. Com 5 minutos de sedimentação, a cor sofreu uma redução de 32,4 uC para 24,3 uC quando adicionado quiabo e a turbidez, de aproximadamente 11,27 uT para 7,5 uT. Em termos de remoção, a cor apresentou melhora de 76,70 para $82,51 \%$ e a turbidez, de 81,76 para $87,82 \%$.

Ainda, em relação aos parâmetros relacionados à matéria orgânica, é possível observar que os valores de UV-254 nm não se diferenciaram significativamente, e houve um aumento do COD com a adição de quiabo como auxiliar de floculação principalmente nos tempos de sedimentação de 15 e 30 min. Com 15 minutos de sedimentação, o COD aumentou de 27,5 para $31,4 \mathrm{mg} \mathrm{L}^{-1}$ 
$(14,1 \%)$ e com 30 minutos, de 29,0 para $33 \mathrm{mg} \mathrm{L}^{-1}(13,7 \%)$. Como esses parâmetros estão relacionados à presença de matéria orgânica, esse fato pode ser justificado pela própria adição de quiabo como auxiliar de floculação. Porém, no tempo considerado ótimo (5 minutos), as variáveis COD e UV-254 nm não se alteraram, o que não prejudica o tratamento, visto que a matéria orgânica adicionada através do auxiliar de floculação é decantada ao final do processo.

\section{CONCLUSÃO}

Nas condições de mistura rápida e lenta utilizadas, o aumento do tempo de sedimentação não proporcionou aumento nas remoções dos parâmetros avaliados, tanto no tratamento em que foi utilizado apenas o coagulante químico Policloreto de Alumínio quanto naquele com adição do auxiliar de floculação Abelmoschus esculentus (L.) Moench (Quiabo), sendo que o tempo de 5 minutos foi suficiente para atingir os melhores resultados.

Em relação ao tratamento com auxiliar de floculação, o aumento da concentração não foi importante para a redução dos parâmetros analisados, visto que a menor concentração proporcionou redução semelhante a de concentrações maiores.

Apesar das variáveis relacionadas à matéria orgânica (COD e UV-254 nm) terem aumentado com a adição de quiabo, nas condições ótimas encontradas, não sofreram alteração, indicando que não houve prejuízo ao tratamento.

Mesmo que os resultados com a adição de quiabo não superem o tratamento realizado com $6 \mathrm{ppm}$ (concentração ótima de PAC), foi observada melhora no tratamento de água para abastecimento. Em comparação com a coagulação/floculação em que se utilizou apenas o coagulante químico a 2 ppm, a adição de quiabo possibilitou maior redução de cor e turbidez, com melhora de, respectivamente, 25 e $33,45 \%$.

\section{REFERÊNCIAS}

BRATBY, J. Coagulation and flocculation in water and wastewater treatment. Londres: IWA publishing, 3 ed. 2016.

CARDOSO, K.C., BERGAMASCO, R., COSSICH, E.S., MORAES, L.C.K. Otimização dos tempos de mistura e decantação no processo de coagulação/floculação da água bruta por meio da Moringa oleífera Lam. Acta

Scientiarum Technology, v. 30, n. 2, p. 415-423, 2008. Disponível em: $<$ https://www.redalyc.org/html/3032/303226522010/>. 10.4025/actascitechnol.v30i2.5493.

CHUNG, C. Y.; SELVAJOO, A.; SETHU, V.; KOYANDE, A.K.; ARPUTHAN, A.; LIM, Z.C. Treatment of palm oil mill effluent (POME) by coagulation flocculation process using peanut-okra and wheat germ-okra. Clean Technologies and Environmental Policy, v. 20, n. 9, p. 1951-1970, 2018. Disponível em: <https://link.springer.com/article/10.1007/s10098-018-1619-y>. doi: 10.1007/s10098018-1619-y.

CRINI, G.; BADOT, P. Application of chitosan, a natural aminopolysaccharide, for dye removal from aqueous solutions by adsorption processes using batch studies: a 
review of recent literature. Progress in polymer science, v. 33, n. 4, p. 399-447, 2008. Disponível em: <https://doi.org/10.1016/j.progpolymsci.2007.11.001>. doi: 10.1016/j.progpolymsci.2007.11.001.

FREITAS, T.K.F.S., OLIVEIRA, V.M., SOUZA, M.T.F., GERALDINO, H.C.L., ALMEIDA, V.C., FÁVARO, S.L., GARCIA, J.C. Optimization of coagulationflocculation process for treatment of industrial textile wastewater using okra (A. esculentus) mucilage as natural coagulant. Industrial Crops and Products, v. 76, p. 538-544, 2015. Disponível em: < https://doi.org/10.1016/j.indcrop.2015.06.027>. doi: 10.1016/j.indcrop.2015.06.027.

LEE, C.S., CHONG, M.F., ROBINSON, J., BINNER, E. Optimization of extraction and sludge dewatering efficiencies of bio-flocculants extracted from Abelmoschus esculentus (okra). Journal of environmental management, v. 157, p. 320-325, 2015.

OLADOJA, N.A. Headway on natural polymeric coagulants in water and wastewater treatment operations. Journal of Water Process Engineering, v. 6, p. 174-192. 2015. Disponível em: < https://doi.org/10.1016/j.jenvman.2015.04.028>. doi: 10.1016/j.jenvman.2015.04.028.

RENAULT, F., SANCEY, B., BADOT, P.M., CRINI, G. Chitosan for coagulation/flocculation processes-an eco-friendly approach. European Polymer Journal, v. $45, \quad$ n. $5, \quad$ p. 1337-1348, 2009. Disponível em: $<$ https://doi.org/10.1016/j.eurpolymj.2008.12.027>. 10.1016/j.eurpolymj.2008.12.027.

SHARMA, B. R.; DHULDHOYA, N. C.; MERCHANT, U. C. Flocculants-an ecofriendly approach. Journal of Polymers and the Environment, v. 14, n. 2, p. 195-202, 2006. Disponível em: <https://link.springer.com/article/10.1007/s10924-0060011-x>. doi: 10.1007/s10924-006-0011-x.

THEODORO, J.D.P., PEREIRA, E.R., VEIT, M.T., BERGAMASCO, R., SANTOS, O.A.A. Estudo da extração salina da semente de Moringa oleífera Lam aplicado na remoção do parâmetro cor para o tratamento de água potável. Revista Tecnológica, p. 275-283, 2015. Disponível em: <http://dx.doi.org/10.4025/revtecnol.v0i0.26090 >. doi: 10.4025/revtecnol.v0i0.26090.

VALVERDE, K.C.; COLDEBELLA, P.F.; BERGAMASCO, R.; Otimização das condições de operação no processo de clarificação de água superficial por meio da associação dos coagulantes moringa oleifera lam e cloreto férrico. Periódico Eletrônico Fórum Ambiental da Alta Paulista, v. 9, n. 11, 2013. Disponível em: <http://dx.doi.org/10.17271/198008279112013557>.

doi: $10.17271 / 198008279112013557$.

YARAHMADI, M., HOSSIENI, M., BINA, B., MAHMOUDIAN, M.H., NAIMABADIE, A., SHAHSAVANI, A. Application of Moringa oleifer seed extract and polyaluminum chloride in water treatment. World Applied Sciences Journal, v. 7, p. 962-967, $2009 . \quad$ Disponível em: $<$ http://citeseerx.ist.psu.edu/viewdoc/download?doi=10.1.1.388.6108\&rep=rep1\&type $=$ pdf>. doi: 10.1 .1 .388 .610 . 